\title{
Research on Performance Seeking Control of Turbofan Engine at Cruise Status Based on Sequential Quadratic Programming Algorithm
}

\author{
Zhao Shufan ${ }^{1, a}$, Wu Heng ${ }^{1}$, Shi Liming ${ }^{2}$, Huang Cheng $^{3}$ and Yang xinyi ${ }^{1}$ \\ ${ }_{1}^{1}$ Aviation Foundation College, Naval Aviation University, Yantai Shandong 264001, China \\ 2 Unit 92198 PLA Force, Huludao Liaoning 125109, China \\ ${ }^{3}$ Unit 78525 PLA Force, Chengdu Sichuan 611630, China
}

\begin{abstract}
The sequential quadratic programming algorithm was applied to the performance seeking control of multi-variable turbofan engine. According to the actual condition of aircraft cruise status, the constant thrust condition is added in the constraint functions of nonlinear programming. Based on the mathematical modes of turbofan engine, the SQP algorithm is adopted to the minimum fuel consumption mode of cruise condition. The simulation results show that after the optimization process, the engine fuel consumption rate decreased by nearly $2 \%$, and the turbine temperature dropped by about $1.2 \%$, the dual optimization benefits have been achieved. There are great potentials in engineering applications.
\end{abstract}

\section{Introduction}

Since the nineties of last century, the aero-engine performance seeking control technology is the latest development of integrated control of aircraft and aero-engine. In traditional design, each component of the aero-engine is reserved for enough margin space, to ensure that can work under the worst working condition. The components designed for the worst working conditions are too conservative to exploit full performance in some normal working conditions. The performance seeking control realtime online estimates the current safety situation, gives full play to the performance of components, and avoids wasting available margin space, to improve one performance or comprehensive performance indicators of aero-engine and to make engine works safely and efficiently.

Aero-engine is a strong nonlinear, multivariable time-varying system. When the nonlinear aeroengine was linearized, it will inevitably result in the conversion error, and the final global optimal solution will deviate from the actual global optimal point. With the development of optimization methods, some non-linear optimization methods are maturing [1]. SQP algorithm is a non-linear optimization method, it can maintain the overall convergence and a local super-convergence at the same time [2]. In this paper, the SQP algorithm was introduced into the aero-engine performance seeking control, which solves the problem of constraint condition and optimization algorithm under the minimum fuel consumption mode. The simulation results were given. The results showed that the SQP method can achieve the global optimal point, make a good optimization effect.

\footnotetext{
${ }^{\mathrm{a}}$ Corresponding author : lightoffreedom@163.com
} 


\section{Sequential Quadratic Programming Algorithm}

For a nonlinear programming problem, it can be expressed as follows.

Objective function: $\min f(x)$

Restrictions: $g(x) \geq 0, h(x)=0$

The main idea of algorithm of SQP is that to solve the problem by changing it to a series of quadratic programming (QP) [3][4][5].

$$
L(x, \lambda)=f(x)+\sum_{i=1}^{m} \lambda_{i} g_{i}(x)
$$

For the general constraint optimization problem, a QP sub-problem can be constructed at the current point $\left(x_{k}, \lambda_{k}\right)$ :

$$
\begin{gathered}
\text { Objective function: } q_{k}(d)=0.5 d^{T} B\left(x_{k}, \lambda_{k}\right) d+\nabla f\left(x_{k}\right)^{T} d \\
\text { Restrictions: } \nabla g_{I}\left(x_{k}\right)^{T} d+g_{I}\left(x_{k}\right) \geq 0, \nabla h_{I}\left(x_{k}\right)^{T} d+h_{I}\left(x_{k}\right)=0
\end{gathered}
$$

The SQP consists of three parts.

First, the Hessian matrix of the Lagrange function is updated in each iteration.

$B$ is the positive definite quasi-newtonian approximation of the Hessian matrix of Lagrange function and is calculated by BFGS method. The iteration equation is:

$$
B_{k+1}=B_{k}+\nabla B_{k}
$$

Where $\nabla B_{k}$ is correction matrix.

Second, the solution of the quadratic program.

A new QP problem will occur in each main iteration of the SQP method.

The target function:

$$
\min \frac{1}{2} x^{T} B x+c^{T} x
$$

Restrictions:

$$
\begin{gathered}
A_{i} x=0, i=1, \ldots, m_{e} \\
A_{i} x \leq b, i=m_{e}+1, \ldots, m
\end{gathered}
$$

Third, one-dimensional search and calculation of target function.

The target function:

$$
\begin{gathered}
\min _{\gamma \in R, c \in R^{n}} \gamma \\
A_{i} x=0, i=1, \ldots, m_{e} \\
A_{i} x-\gamma \leq b, i=m_{e}+1, \ldots, m
\end{gathered}
$$

The calculation flow was shown in figure 1 . 


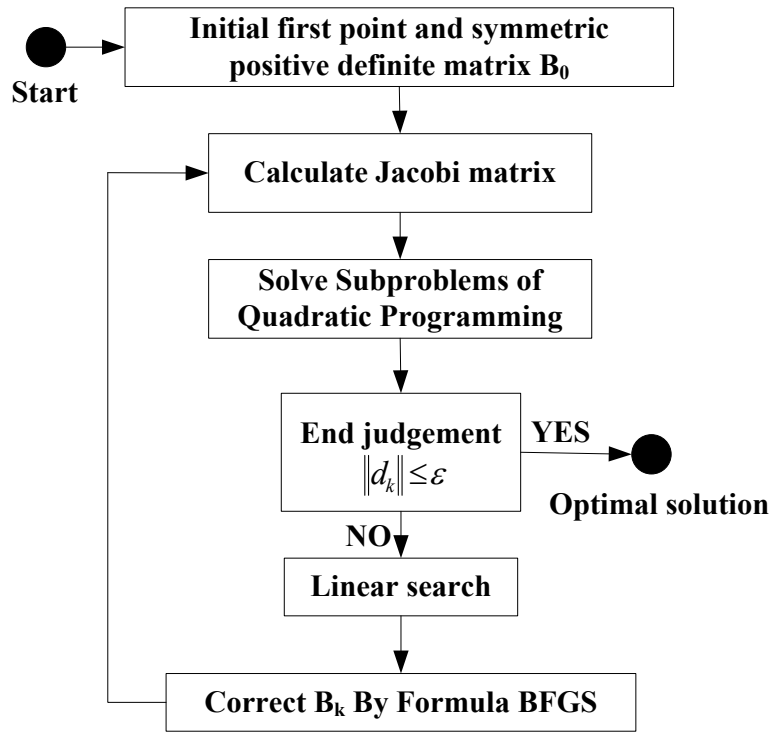

Figure 1. Calculation process of SQP algorithm

\section{Engine Performance Seeking Control Mode}

Integrated control system based on the aero-engine performance seeking control technology is shown in figure 2, the system is a typical upper and lower computer structure. The upper computer is a closed loop real-time simulation system which mainly has the flight controller, the rudder surface mechanism, the aircraft and aero-engine, which mainly gives the flight control instruction, the aero-engine control instruction and the aero-engine optimization control mode. The lower computer is mainly the calculation module, which is composed of the aero-engine airborne real-time mode, the mode selector and the optimization calculation module. The upper computer gives the flight status information and the optimization control mode command to the lower computer. The lower computer optimizes the control law by the airborne mode and the optimization calculation module, and then gives it to digital electronic controller to obtain a more favorable thrust response.

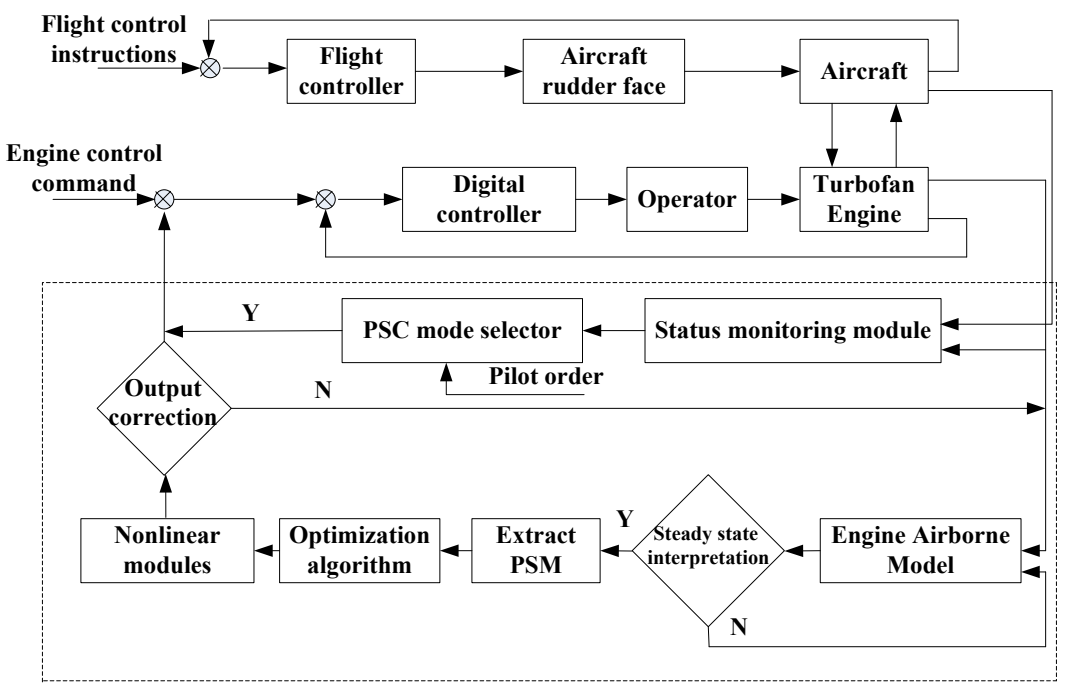

Figure 2. Comprehensive control of aircraft and engine 
The relationship between the control variable and the state variable is $X=P u$. The performance seeking control technique optimizes the control variable $u$ by the onboard engine mode to optimize one parameter or the combination of several parameters of state parameter $X$. Therefore, the performance seeking control technology is expressed as a non-linear programming form.

$$
\begin{gathered}
\text { Performance: } \max (\min ) f(u) \\
\text { Restrictions: } g_{i, \min } \leq G_{i}(u) \leq g_{i, \max }, i=0,1,2 \ldots
\end{gathered}
$$

In which $G_{i}(u)$ are restriction, usually include: the range of control variable, the limit position of the control structure, the safety limit to ensure the normal operation of the engine, such as no overheating, no over-rev and so on.

The actual work of the engine is subject to the following constraints: high and low pressure rotor speed limit, fan and compressor surge margin limit, maximum fuel supply limit and its rate of change, turbine temperature limit, compressor guide blade angle restrictions and its rate of change restrictions. The constraint function is normalized to avoid the calculation error due to the different dimension of each constraint function.

$$
g_{i}=\left|u_{i} / u_{i, \max }\right|-1 \leq 0
$$

Performance seeking control mode is closely related to the mission, the demand of different tasks on engine is different. There are three main modes, the maximum thrust mode, the minimum fuel consumption mode and the lowest turbine temperature mode.

The minimum fuel consumption mode is mainly used for aircraft steady-state flight process. Relevant data shows that for a normal routine flight mission, there are nearly $85 \%$ of the time and $45 \%$ of the fuel spent on the cruise process. By optimizing control law of the engine in the minimum fuel consumption mode, the fuel consumption of the engine will reduce while the engine thrust meets the needs of steady-state flight. It will help improving the flight performance of aircraft. Of course, the mode must ensure that the engine work safely, otherwise there is no practical significance. The corresponding non-linear programming is:

$$
\begin{aligned}
& \min s f c \\
& w f_{\min } \leq W F \leq w f_{\max } \\
& A 8_{\min } \leq A 8 \leq A 8_{\max } \\
& p n f_{\min } \leq P N F \leq p n f_{\max } \\
& \text { pnc }_{\min } \leq P N C \leq p n c_{\max } \\
& t_{5 \min } \leq T_{5} \leq t_{5 \max } \\
& F=\text { const }
\end{aligned}
$$

Where $s f c$ is specific fuel consumption, $W F$ is fuel flow of combustor, $A 8$ is outlet area of nozzle, $P N F$ is relative speed of fan, $P N C$ is relative speed of compressor, $T_{5}$ is outlet temperature of low pressure turbine.

\section{Simulation Analysis of Turbofan Engine Performance Seeking Control}

\subsection{Analysis of Thrust Need}

In the engine performance seeking control technology, the minimum fuel consumption mode is much more difficult than the maximum thrust mode. There is a strong equality constraint in the constraint condition. It becomes more difficult [6] from the algorithm design or running time. On the other hand, the relevant research is optimized for the control law [7], either the maximum thrust mode [8] at the fixed height, the speed, or the minimum fuel consumption mode at the fixed speed [9].

Before the optimization of the control law in minimum fuel consumption, the thrust demand $F_{R}$ of the current flight condition should be obtained according to the actual task demand of the aircraft, so 
$F=F_{R}$. This ensures that the turbofan engine produces the corresponding thrust under the current control law and the thrust can match the actual mission requirements.

\subsection{Minimum fuel consumption mode of aircraft cruise}

Flight conditions were flying height $11000 \mathrm{~m}$, flight speed $0.5 \mathrm{Ma}$, engine state parameters at cruise flat flight are: $X=\{F, s f c, P N C, P N F, T \cdots\}$, engine control variables are: $u=\left\{W F, A_{8}, d G v c, d G v f\right\}, d G v c$ is angle of compressor guide vane, $d G v f$ is angle of fan guide vane.

Objective function: engine performance parameters $\min s f c$

Equality Constraints: the engine power of the state is satisfied $F_{R}=2005 \mathrm{daN}$

Inequality constraints:

$$
\begin{aligned}
& w f_{\min } \leq W F \leq w f_{\max } \\
& A 8_{\text {min }} \leq A 8 \leq A 8_{\max } \\
& d G v f_{\min } \leq d G v f \leq d G v f_{\max } \\
& d G v c_{\min } \leq d G v c \leq d G v c_{\max } \\
& p n f_{\min } \leq P N F \leq p n f_{\max } \\
& p n c_{\min } \leq P N C \leq p n c_{\max } \\
& t_{5 \min } \leq T_{5} \leq t_{5 \max } \\
& S M L \geq S M L_{\min }
\end{aligned}
$$

Where $S M L$ is surge margin of low pressure. The status and control variable changes of turbofan engine performance seeking control were shown in figure 3.

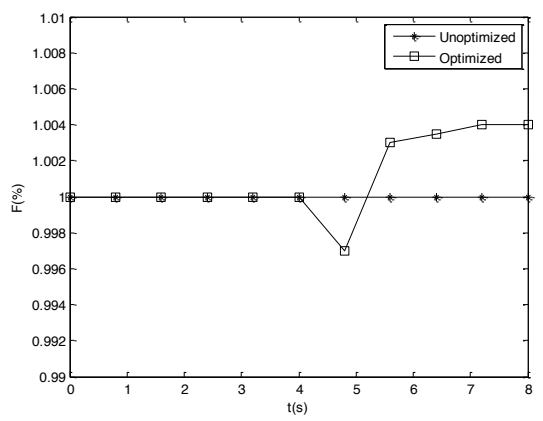

(a) F in the PSC process

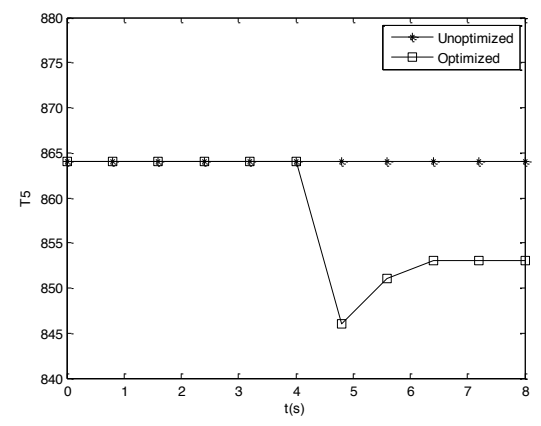

(c) T5 in the PSC process

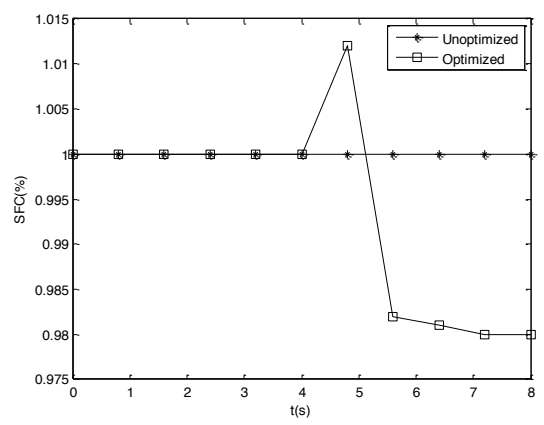

(b) SFC in the PSC process

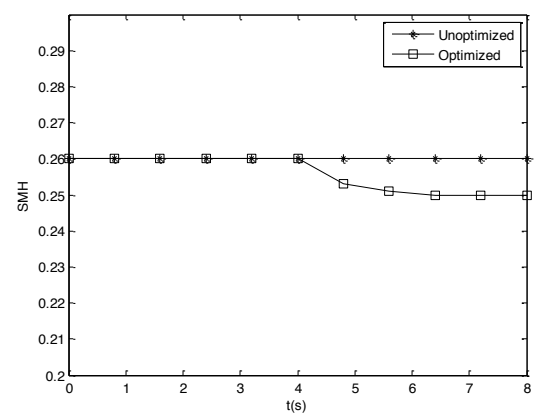

(d) SMH in the PSC process 


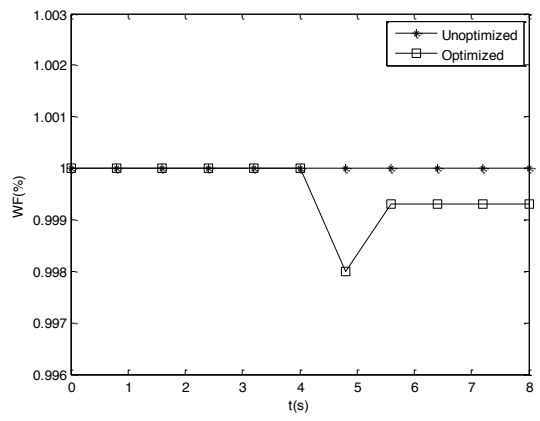

(e) WF in the PSC process

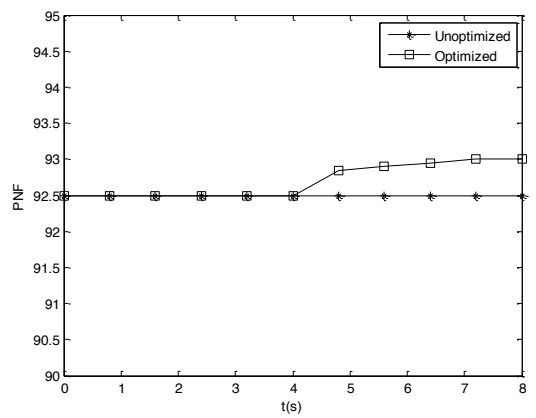

(g) PNF in the PSC process

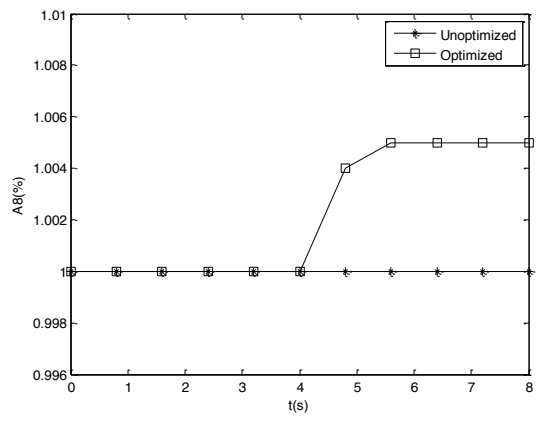

(f) A8 in the PSC process

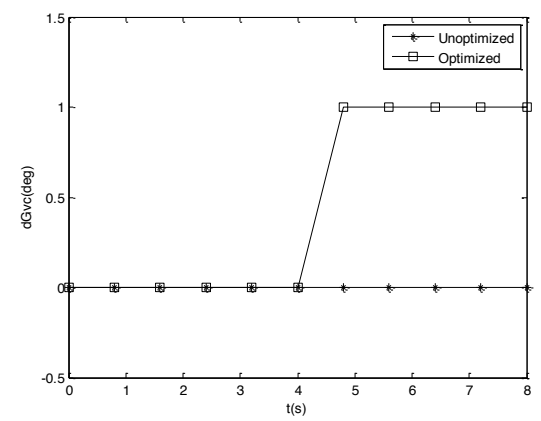

(h) dGvc in the PSC process

Figure 3. Curve of engine PSC process of aircraft cruise

Due to existence of the equation constraints of thrust constant, the simulation time is longer. The control variables start changing at fourth second, the minimum fuel consumption mode reduces the main fuel flow, changes the nozzle area and angle of guide vane to achieve control goal. The trends of control variables change are in line with the principle of the mode, and it tends to be stable after 6 seconds. After the optimization process, the engine thrust increased slightly, the fuel consumption decreased by nearly $2 \%$, the rotor system speed increased slightly, and the turbine temperature dropped by about $1.2 \%$, the dual optimization benefits have been achieved.

\section{Conclusion}

The results showed that the addition of constant thrust condition conforms to the actual situation of aircraft cruising condition, and the application of SQP algorithm in performance seeking control of multi-variable turbofan engine is feasible. It can reduce the fuel consumption rate and the turbine temperature while keeping the thrust of the engine unchanged. The dual optimization benefits have been achieved. There are great potentials in engineering applications.

\section{Acknowledgments}

This work is supported by special funds of Taishan Scholar Project, National Natural Science Fund (51505492) and Natural Science Foundation of Shandong Province(ZR2016FQ19).

\section{References}

1. Matthew C T, Declan G B and Ian P. 2001 Input/Output Conditioning of Robust Integrated Flight/Propulsion Control Journal of Guidance Control and Dynamics, p 6 
2. James W D 2002 Integrated Flight and Propulsion Control: Current Successes and Remaining Challenges AIAA p 6021

3. Wang R X 2011 Research on Modeling and Control of Flight / Push Integrated System (Nanjing: Nanjing University of Aeronautics and Astronautics) p 20

4. Yuan Y X and Sun W Y 2001 Optimization theory and method (Beijing: Beijing Science and Technology Press) p 33

5. Liu Y, Tang L S and Chen Y P 1996 Nonlinear parallel algorithm genetic algorithm (Beijing: Beijing Science and Technology Press) p 22

6. Sun F C 2009 Research on Optimal Control Technology of Aeroengine Performance (Nanjing: Nanjing University of Aeronautics and Astronautics) p 15

7. Wu D 2004 Research on Nonlinear Performance Optimization of Aviation Propulsion System (Xian: Northwestern Polytechnical University) p 50

8. Li P Z 2007 Application of Genetic Algorithm in Cruise Optimization Control of Turbofan Engine (Xian: Northwestern Polytechnical University) p 29

9. Liu X D 2007 Research on Performance Optimization of Turbofan Engine Based on Genetic Algorithm (Xian: Northwestern Polytechnical University) p 37 